\title{
An Explicit Construction of a Class of Suspensions and Autonomous Differential Equations for Diffeomorphisms in the Plane
}

\author{
Gottfried Mayer-Kress ${ }^{1}$ and Hermann Haken ${ }^{2}$ \\ 1 Center for Nonlinear Studies and Theoretical Division, Los Alamos National Laboratory, \\ Los Alamos, NM 87545, USA \\ ${ }^{2}$ Institut für Theoretische Physik, Universität Stuttgart, D-7000 Stuttgart 80, \\ Federal Republic of Germany
}

\begin{abstract}
From a large class of diffeomorphisms in the plane, which are known to produce chaotic dynamics, we explicitly construct their continuous suspension on a three dimensional cylinder. This suspension is smooth $\left(C^{1}\right)$ and can be characterized by the choice of two smooth functions on the unit interval, which have to fulfill certain boundary conditions. For the case of entire Cremona transformations, we are able to construct the corresponding autonomous differential equations of the flow explicitly. Thus it is possible to relate properties of discrete maps to those of ordinary differential equations in a quantitative manner. Furthermore, our construction makes it possible to study the exact solutions of chaotic differential-equations directly.
\end{abstract}

\section{Introduction}

For the description of dynamical long time behavior of complex systems, two kinds of models have been studied in the literature. The classical models are based on ordinary differential equations, which means that a continuous flow of time is considered. More recently models with discrete time-steps $\tau$ have become popular, especially for the description of erratic or chaotic behavior [1]. In this way, the frequencies of the dynamical system above a maximal frequency $\omega_{\max }=\frac{2 \pi}{\tau}$ are neglected. These models are based on ordinary difference equations and have been successful in modelling different routes to turbulence [2]. In a general sense it is clear that both of these approaches are equivalent in the description of the different transitions that occur before chaotic behavior sets in. However, little is known about how a given property of a discrete model can be translated into a continuous model and vice versa. For instance it is known for a unimodal map on the interval that the order of its maximum determines its universality class in the period-doubling route to chaos [3]. But the corresponding criterion for differential equations is not clear [4]. The main geometrical argument used in this work is based on the concept of Poincaré-maps [5]. This is a 
geometrical method by which one can construct a discrete dynamical model from a recurrent continuous flow. Although it is now a standard method for the numerical analysis of chaotic flows to construct the corresponding Poincaré-map, there are only very few examples [6] in which this Poincaré-map can be given explicitly. On the other hand, similar difficulties are met if a differential equation should be associated with a given discrete map. Since discrete dynamical systems possess a natural high-frequency cut-off at $\omega_{\max }$, the construction of a continuous time suspension is by no means unique [13]. Therefore we will construct a continuous flow, which should come as close as possible to a damped and driven anharmonic oscillator. This is the natural analogue of an entire Cremona transformation because of the position independent damping factor.

In the second section we shall introduce the basic notions, then we shall show in the third section how a suspension is constructed [see Eq. (3.2)] from a given family of interpolating diffeomorphisms in the plane. In Sect. 4 we shall construct a general interpolating family of diffeomorphisms [see Eq. (4.2)]. We shall restrict ourselves to entire Cremona transformations in Sect. 5 [Eq. (5.5)], where we also shall select a specific realization [Eq. (5.12)]. Finally we shall write down the explicit autonomous differential equations for this system in Sect. 6 [Eq. (6.3)].

\section{Basic Notions}

In this section we relate discrete time dynamics in the plane with continuous dynamics in the three dimensional space. Thus let $T: \mathbb{R}^{2} \rightarrow \mathbb{R}^{2}$ be a diffeomorphism in the plane, i.e., $T$ is a smooth, invertible map, with a smooth inverse $T^{-1}$. A discrete trajectory $\left(x_{n}, y_{n}\right)$ is created by $T$ via:

$$
\left(x_{n+1}, y_{n+1}\right)=T\left(x_{n}, y_{n}\right),
$$

where $n \in \mathbb{Z}$ represents the discrete time-variable. Continuous time dynamical systems, which are defined by differential equations, generate trajectories of a flow which is defined by:

Definition 2.1. Let $M_{0} \subset \mathbb{R}^{3}$ be some smooth manifold, and let $\operatorname{Diff}\left(M_{0}\right)$ be the space of all diffeomorphisms on $M_{0}$. Then a mapping $\phi: \mathbb{R} \rightarrow \operatorname{Diff}\left(M_{0}\right), t \mapsto \phi_{t}$ is called a (continuous) flow on $M_{0}$ iff:

(i) $\phi_{0}=\mathrm{id}$,

(ii) $\phi_{s+t}=\phi_{s} \circ \phi_{t}$ for $s, t \in \mathbb{R}$.

Here "id" is the identity mapping and "o" denotes composition of maps. In a physical language $\phi_{t}$ shifts some initial value $\mathbf{x}_{0} \in M_{0}$ along its trajectory up to the point $\phi_{t}\left(\mathbf{x}_{0}\right)=: \mathbf{x}(t)$, which corresponds to a time-interval of length $t$. In his fundamental paper [7], Smale described how a continuous flow can be constructed as a suspension of some diffeomorphism. To this end we have (cf. [8]):

Definition 2.2. Let $M_{0} \subset \mathbb{R}^{3}$ be a smooth manifold with closed subset $S \subset M_{0}$. Let $T: S \rightarrow S$ be a diffeomorphism on $S$ and $\phi$ a flow on $M_{0}$. If there exist $\alpha, \beta \in \mathbb{R}^{+}$such that:

$$
\bigcup_{t \in(0, \alpha)} \phi_{t}(S)=M_{0}
$$




$$
\bigcup_{t \in(0, \beta)} \phi_{t}(S)=: S_{\beta} \subset M_{0} \text { open such that: } S_{\beta} \cap S=\phi \text {, }
$$

$$
\left.\phi_{\beta}\right|_{S}=T,
$$

then $\phi$ is called a constant time suspension of $T$.

The diffeomorphism $T$ is then called the Poincaré-map of the flow $\phi$.

Statement (iii) means that for the return time $\beta$, which determines the maximal frequency resolved by $T$, the restriction of the diffeomorphism $\phi_{\beta} \in \operatorname{Diff}\left(M_{0}\right)$ to the subsurface $S$ coincides with the diffeomorphism $T$ on $S$. The construction of Smale [7] is based on the manifold $M_{T}$, which is defined by:

$$
M_{T}:=\left\{(x, y, u) \in \mathbb{R}^{3} ;(x, y, u+1) \sim(T(x, y), u)\right\},
$$

where " $\sim$ " means that the corresponding points are identified. The variable $u$ corresponds to a "phase angle" and $M_{T}$ can be thought of as some "generalized Möbius-strip." The suspension $\tilde{\phi}$ on $M_{T}$ can now be defined as:

$$
\tilde{\phi}_{t}(x, y, u):=(x, y, u+t) \text {. }
$$

We can see that for a cross-section $S \subset M_{T}$ which is given by

$$
S:=\left\{(x, y, u) \in M_{T}: u=0\right\},
$$

the suspension $\tilde{\phi}$ reproduces $T$ in the sense that: $\left.\tilde{\phi}_{1}\right|_{s}=T$, i.e. $\tilde{\phi}_{1}(x, y, 0)$ $=(T(x, y), 0)$. From the construction of the suspension described above it becomes clear that all of the dynamics of the system are built into the structure of the manifold $M_{T}$ such that the explicit structure of $\tilde{\phi}$ appears to become trivial.

In the following we will use an approach which is physically more intuitive. Instead of the manifold $M_{T}$ we consider the cylinder $M$ defined by:

$$
M:=\left\{(x, y, u) \in \mathbb{R}^{3} ;(x, y, u+1) \sim(x, y, u)\right\} .
$$

That means $u$ is a cyclic variable which can be interpreted as a phase-angle, e.g. of some periodic driving force. This interpretation conforms to our motivation for this work, which is to model a periodically driven damped anharmonic oscillator starting from a discrete two-dimensional map.

\section{Construction of the Suspension from a Given Family of Interpolating Diffeomorphisms}

\subsection{Continuous Suspension}

In this section we suspend the diffeomorphism $T$ of the plane (see Sect. 2). To this end we introduce a family of interpolating diffeomorphisms $F_{t} \in \operatorname{Diff}\left(\mathbb{R}^{2}\right)$. In Sects. 4 and 5 we shall construct explicit examples for $F_{t}$.

Definition 3.1. Let $F_{t}: \mathbb{R}^{2} \rightarrow \mathbb{R}^{2}$ be a diffeomorphism for all $t \in \mathbb{R}$ such that:

$$
\begin{gathered}
F_{0}=\mathrm{id}, \\
F_{1}=T, \\
F_{t}=F_{\varepsilon} \circ T^{n} \quad \text { if } \quad t=n+\varepsilon>1, n \in \mathbb{Z},
\end{gathered}
$$


where we have used the abbreviation $T^{n}:=T \circ T^{n-1}, T^{0}:=$ id. If $F_{t}$ depends smoothly on the time-parameter $t$, we call it an interpolating diffeomorphism for $T$.

The requirement of smooth dependence of $t$ implies that the Jacobiandeterminant of $F_{t}$ also has to be a smooth function of $t$. This fact is important because diffeomorphisms $\hat{T}$ with negative Jacobian cannot be suspended and therefore cannot be considered as the Poincare-map of some continuous flow. Otherwise there had to exist a $t^{*} \in(0,1)$ for which the Jacobian of $F_{t^{*}}$ would vanish. This is because of the fact that $F_{0}=\mathrm{id}$, which implies: $\operatorname{det} F_{0}=1>0>\operatorname{det} F_{1}=\operatorname{det} \hat{T}$ and because of the continuity of $\operatorname{det} F_{t}$ as a function of $t$. This means that $F_{t^{*}}$ is not a diffeomorphism, which is in contradiction to the Definition 3.1.

We now can construct a class of suspensions for a given diffeomorphism $T$ in the plane by the following:

Proposition 3.1. Let $M$ be the cylinder defined in (2.5) and let $\Sigma$ be the cross-section defined by

$$
\Sigma:=\{(x, y, u) \in M, u=0\} .
$$

Let $F_{t}$ be an interpolating diffeomorphism for a given diffeomorphism $T$ defined on $\Sigma$, where $t=n+\varepsilon \in \mathbb{R}, n \in \mathbb{Z}, \varepsilon \in[0,1)$. Then the mapping $\phi: \mathbb{R} \rightarrow \operatorname{Diff} M, t \mapsto \phi_{t}$ defined by

$$
\phi_{t}(x, y, u):=\left(F_{u+\varepsilon} \circ T^{n} \circ F_{u}^{-1}(x, y), u+\varepsilon\right)
$$

is a constant time suspension of $T$.

Remark. We identify the family of cross sections $\phi_{t}(\Sigma) \subset M$ with the plane $\mathbb{R}^{2}$.

Proof of Proposition 3.1. First we have to show that $\phi$ describes a flow. Property (i) of Definition 2.7 can be easily checked by insertion. For part (ii) we have to distinguish between different cases.

1) Case. $\phi_{n+\varepsilon}=\phi_{n} \circ \phi_{\varepsilon}$. From (3.2) we get

$$
\phi_{n+\varepsilon}(x, y, u)=\left(F_{u+\varepsilon} \circ T^{n} \circ F_{u}^{-1}(x, y), u+\varepsilon\right) .
$$

Now we insert the identity id $=F_{u+\varepsilon}^{-1} \circ F_{u+\varepsilon}$, and obtain

$$
\begin{aligned}
\phi_{n+\varepsilon}(x, y, u) & =\left(F_{u+\varepsilon} \circ T^{n} \circ F_{u+\varepsilon}^{-1} F_{u+\varepsilon} \circ F_{u}^{-1}(x, y), u+\varepsilon\right) \\
& =\phi_{n}\left(F_{u+\varepsilon} \circ F_{u}^{-1}(x, y), u+\varepsilon\right)=\phi_{n} \circ \phi_{\varepsilon}(x, y, u) .
\end{aligned}
$$

Because of the asymmetrical role of $n$ and $\varepsilon$ we show the

2) Case. $\phi_{n+\varepsilon}=\phi_{\varepsilon} \circ \phi_{n}$. With the same argument as above we get:

$$
\begin{aligned}
\phi_{n+\varepsilon}(x, y, u) & =\left(F_{u+\varepsilon} \circ T^{n} \circ F_{u}^{-1}(x, y), u+\varepsilon\right)=\left(F_{u+\varepsilon} \circ F_{u}^{-1} \circ F_{u} \circ T^{n} \circ F_{u}^{-1}(x, y), u+\varepsilon\right) \\
& =\phi_{\varepsilon}\left(F_{u} \circ T^{n} \circ F_{u}^{-1}(x, y), u\right)=\phi_{\varepsilon} \circ \phi_{n}(x, y, u) .
\end{aligned}
$$

Furthermore we have to show the

3) Case. $\phi_{n+m}=\phi_{n} \circ \phi_{m}$. This is done by:

$$
\begin{aligned}
\phi_{n+m}(x, y, u) & =\left(F_{u} \circ T^{n+m} \circ F_{u}^{-1}(x, y), u\right) \\
& =\left(F_{u} \circ T^{n} \circ F_{u}^{-1} \circ F_{u} \circ T^{m} \circ F_{u}^{-1}(x, y), u\right) \\
& =\phi_{n}\left(F_{u} \circ T^{m} \circ F_{u}^{-1}(x, y), u\right)=\phi_{n} \circ \phi_{m}(x, y, u) .
\end{aligned}
$$


Finally we have to look at the

4) Case. $\phi_{\varepsilon+\delta}=\phi_{\varepsilon} \circ \phi_{\delta}$. Here we have:

$$
\begin{aligned}
\phi_{\varepsilon+\delta}(x, y, u) & =\left(F_{\varepsilon+\delta+u} \circ F_{u}^{-1}(x, y), u+\varepsilon+\delta\right) \\
& =\left(F_{\varepsilon+\delta+u} \circ F_{u+\varepsilon}^{-1} \circ F_{u+\varepsilon} \circ F_{u}^{-1}(x, y), u+\varepsilon+\delta\right) \\
& =\phi_{\delta}\left(F_{u+\varepsilon} \circ F_{u}^{-1}(x, y), u+\varepsilon\right)=\phi_{\delta} \circ \phi_{\varepsilon}(x, y, u) .
\end{aligned}
$$

For the case that one of the above sums, which appear as indices of $F_{1}$, becomes larger than one, we apply property (iii) of Definition 3.7. The conditions (i) and (ii) of Definition 2.2 are obviously satisfied by $\phi$, and property (iii) can be seen by noting that

$$
F_{0}=F_{0}^{-1}=\mathrm{id}
$$

Thus we have arrived at a prescription which allows us to obtain a system of trajectories from $T$ which depend continuously on the time-parameter $t$. In the next subsection we shall formulate conditions which will guarantee that these trajectories are also smooth. This smoothness is necessary for the trajectories to arise from differential equations.

\subsection{Differentiable Suspension}

The next step in our procedure will be to examine under which conditions the timederivative of the flow $\phi_{t}$ is a continuous function of $t$. From the definition in (3.2) and the linearity of the differentiation-operation we can see that besides the trivial time-dependence of the $u$-component of $\phi_{t}$ only the function $F_{u+\varepsilon}$ will be affected. This means that we only have to require the differentiability of $F_{t}$ with respect to $t$. For $t=n+\varepsilon$ we obtain:

$$
\frac{d \phi_{t}}{d t}(x, y, u)=\left(\frac{d F_{u+\varepsilon}}{d \varepsilon}(X, Y), 1\right),
$$

where $(X, Y):=T^{n} \circ F_{u}^{-1}(x, y)$.

Thus we have to make sure that $d F_{u+\varepsilon} / d \varepsilon$ is continuous for all $(X, Y)$. To make this condition explicit, we may choose as initial condition a point which lies in $\Sigma$. Then Eq. (3.3) will be replaced by:

$$
\frac{d \phi_{t}}{d t}(x, y, 0)=\left(\frac{d F_{\varepsilon}}{d \varepsilon}\left(T^{n}(x, y)\right), 1\right) .
$$

Note that this differential equation is not autonomous, since it depends explicitly on the time variable $t=\varepsilon$ and on the initial conditions $(x, y) \in \Sigma$. Equation (3.4) represents a system of trajectories which are smooth in the open interval $(0,1)$ and, according to Definition (3.2), also are smooth in every open interval $(n, n+1)$. We now have to formulate the condition that the system passes smoothly through the 
Poincaré-sections at $t=n$. Thus we have to consider the two one-sided limits:

$$
\begin{aligned}
& \lim _{t \rightarrow 1_{-}} \frac{d \phi_{t}}{d t}(x, y, 0)=\lim _{\varepsilon \rightarrow 1-}\left(\frac{d F_{\varepsilon}}{d \varepsilon}(x, y), 1\right), \\
& \lim _{t \rightarrow 1_{+}} \frac{d \phi_{t}}{d t}(x, y, 0)=\lim _{\varepsilon \rightarrow 0_{+}}\left(\frac{d F_{\varepsilon}}{d \varepsilon}(T(x, y)), 1\right) .
\end{aligned}
$$

The requirement that the limits coincide is a continuity condition for the derivative of the interpolating diffeomorphism $F_{\varepsilon}$.

\section{Construction of an Interpolating Family for Arbitrary Diffeomorphisms in the Plane}

In this section we shall state the requirements which a class of maps on the interval have to fulfill in order to belong to our family of interpolating diffeomorphisms. We state our result in the following:

Proposition 4.1. Let $\xi_{i k} \in \mathscr{C}^{1}(I)$ be smooth functions on the unit interval for $i, k \in\{1,2\}$. Let the following set of conditions hold:

$$
\begin{aligned}
& \xi_{i 1}(0)=\xi_{i 2}(1)=1, \\
& \xi_{i 1}(1)=\xi_{i 2}(0)=0, \\
& \dot{\xi}_{i 1}(0)=\dot{\xi}_{i 2}(1), \\
& \dot{\xi}_{i 1}(1)=\dot{\xi}_{i 2}(0)=0 .
\end{aligned}
$$

where

$$
\dot{\xi}_{i, k}:=\frac{d \xi_{i, k}}{d t}
$$

Let $T=\left(\begin{array}{l}f_{1} \\ f_{2}\end{array}\right)$ be a diffeomorphism in the plane with components $f_{i}$, such that for all points $\left(\begin{array}{l}x \\ y\end{array}\right)$ and all $t \in I$, we have

$$
\xi_{11}(t)\left(\xi_{21}(t)+\xi_{22}(t) \partial_{y} f_{2}\left(\begin{array}{l}
x \\
y
\end{array}\right)\right)+\xi_{12}(t)\left(\xi_{21}(t) \partial_{x} f_{1}\left(\begin{array}{l}
x \\
y
\end{array}\right)+\xi_{22}(t) \operatorname{det} J_{T}\left(\begin{array}{l}
x \\
y
\end{array}\right)\right)>0
$$

Then a family $F_{t}$ of interpolating diffeomorphisms is given by

$$
F_{t}\left(\begin{array}{l}
x \\
y
\end{array}\right)=\left(\begin{array}{c}
x \xi_{11}(t)+f_{1}\left(\begin{array}{l}
x \\
y
\end{array}\right) \xi_{12}(t) \\
y \xi_{21}(t)+f_{2}\left(\begin{array}{l}
x \\
y
\end{array}\right) \xi_{22}(t)
\end{array}\right) .
$$

The induced suspension $\phi_{t}$ is differentiable.

Proof. Inserting conditions (i) and (ii) into (4.3) yields the interpolating properties of $F_{t}$ (see Definition 3.1). The condition (4.2) guarantees that for all $t \in I$ the map $F_{t}$ 
is a diffeomorphism, since the Jacobian of $F_{t}$ is given by:

$$
J_{F_{t}}\left(\begin{array}{l}
x \\
y
\end{array}\right)=\left(\begin{array}{cc}
\xi_{11}(t)+\xi_{12}(t) \partial_{x} f_{1}\left(\begin{array}{l}
x \\
y
\end{array}\right) & \xi_{12}(t) \partial_{y} f_{1}\left(\begin{array}{l}
x \\
y
\end{array}\right) \\
\xi_{22}(t) \partial_{x} f_{2}\left(\begin{array}{l}
x \\
y
\end{array}\right) & \xi_{21}(t)+\xi_{22}(t) \partial_{y} f_{2}\left(\begin{array}{l}
x \\
y
\end{array}\right)
\end{array}\right)
$$

and therefore, by omitting the arguments we have:

$$
\begin{aligned}
\operatorname{det} J_{F}\left(\begin{array}{l}
x \\
y
\end{array}\right)= & \xi_{11} \xi_{21}+\xi_{11} \xi_{22} \partial_{y} f_{2}+\xi_{12} \xi_{21} \partial_{x} f_{1} \\
& +\xi_{11} \xi_{22}\left(\partial_{x} f_{1} \partial_{y} f_{2}-\partial_{x} f_{2} \partial_{y} f_{1}\right),
\end{aligned}
$$

which can be simplified to yield the expression (4.2). In order to show that the suspension we have obtained is also differentiable, we consider the time derivative of $F_{t}$. It is given by

$$
\dot{F}_{t}\left(\begin{array}{l}
x \\
y
\end{array}\right)=\left(\begin{array}{c}
x \dot{\xi}_{11}(t)+\dot{\xi}_{12}(t) f_{1}\left(\begin{array}{l}
x \\
y
\end{array}\right) \\
y \dot{\xi}_{21}(t)+\dot{\xi}_{22}(t) f_{2}\left(\begin{array}{l}
x \\
y
\end{array}\right)
\end{array}\right)
$$
since the functions $f_{i}$ are time-independent. The continuity-condition of $\dot{F}_{t}\left(\begin{array}{l}x \\ y\end{array}\right)$ at
the Poincaré-section $t=1$ is expressed by the equations:

$$
\lim _{t \rightarrow 1-}\left(\begin{array}{c}
x \dot{\xi}_{11}(t)+\dot{\xi}_{12}(t) f_{1}\left(\begin{array}{l}
x \\
y
\end{array}\right) \\
y \dot{\xi}_{21}(t)+\dot{\xi}_{22}(t) f_{2}\left(\begin{array}{l}
x \\
y
\end{array}\right)
\end{array}\right)=\lim _{\varepsilon \rightarrow 0+}\left(\begin{array}{l}
\dot{\xi}_{11}(\varepsilon) f_{1}\left(\begin{array}{l}
x \\
y
\end{array}\right)+\dot{\xi}_{12}(\varepsilon) f_{1}\left(T\left(\begin{array}{l}
x \\
y
\end{array}\right)\right) \\
\dot{\xi}_{21}(\varepsilon) f_{2}\left(\begin{array}{l}
x \\
y
\end{array}\right)+\dot{\xi}_{22}(\varepsilon) f_{2}\left(T\left(\begin{array}{l}
x \\
y
\end{array}\right)\right)
\end{array}\right) .
$$

Performing the limits and inserting the conditions (iii) and (iv) shows that this equation holds. Thus we have shown that the induced diffeomorphism is indeed differentiable.

When we choose the Hénon-map as the diffeomorphism to be suspended according to Eq. (4.2) we observe that the divergence of the induced flow is not position independent and therefore cannot be interpreted as belonging to a driven damped oscillator. Therefore we are going to introduce a different class of suspensions, which conserve the properties of entire Cremona transformations, namely to possess position-independent dissipation rates.

\section{Suspensions of Entire Cremona Transformations}

In this section we will consider diffeomorphisms in the plane with a constant Jacobian. They can be written in the form:

$$
T\left(\begin{array}{l}
x \\
y
\end{array}\right)=\left(\begin{array}{c}
f_{a}(x)+y \\
-j x
\end{array}\right)
$$


where $j>0$ and $f_{a}(x)$ is a nonlinear function on the real line with $a \in \mathbb{R}$ as the relevant parameter. Note that for the case $f_{a}(x)=1-a x^{2}$ the diffeomorphism $T$ corresponds to the Hénon-system [9], which is well studied for its chaotic aspects. It is also among the simplest models which create a "horse-shoe" in the plane (cf. [8]). The Jacobian of $T$ is simply given by

where

$$
\operatorname{det} J_{T}\left(\begin{array}{l}
x \\
y
\end{array}\right)=j,
$$

$$
J_{T}\left(\begin{array}{l}
x \\
y
\end{array}\right)=\left(\begin{array}{cc}
f^{\prime}(x) & 1 \\
-j & 0
\end{array}\right)
$$

The interpolating family of diffeomorphisms $F_{t}$ for a suspension of $T$ is given in the following proposition:

Proposition 5.1. Let $\xi, \eta \in \mathscr{C}^{1}([0,1])$ such that $\xi(t)^{2}+\eta(t)^{2}>0$ for all $t \in[0,1]$. Assume that the following boundary conditions are satisfied by $\xi$ and $\eta$ :

$$
\begin{aligned}
& \xi(0)=\eta(1)=\dot{\xi}(0)=\dot{\xi}(1)=\dot{\eta}(1)=0, \\
& \xi(1)=\eta(0)=1, \\
& \dot{\eta}(0)=\ln j \quad \text { for } \quad j>0 .
\end{aligned}
$$

Furthermore let $\zeta(t)=\frac{j^{t}}{\xi(t)^{2}+\eta(t)^{2}}$, then we have an interpolating family of diffeomorphisms for the entire Cremona-transformation $T$ of (5.1) given by

$$
F_{t}\left(\begin{array}{c}
x_{0} \\
y_{0}
\end{array}\right)=\left(\begin{array}{c}
\eta(t) \zeta(t) x_{0}+\xi(t)^{2} f\left(x_{0}\right)+\xi(t) y_{0} \\
-\xi(t) \zeta(t) x_{0}+\xi(t) \eta(t) f\left(x_{0}\right)+\eta(t) y_{0}
\end{array}\right)
$$

Proof. The Jacobian matrix of $F_{t}$ is given by:

$$
J_{F_{t}}\left(\begin{array}{l}
x_{0} \\
y_{0}
\end{array}\right)=\left(\begin{array}{cc}
\eta(t) \zeta(t)+\xi(t)^{2} f^{\prime}\left(x_{0}\right) & \xi(t) \\
-\xi(t) \zeta(t)+\xi(t) \eta(t) f^{\prime}\left(x_{0}\right) & \eta(t)
\end{array}\right) .
$$

We obtain for its determinant the expression:

$$
\begin{aligned}
\operatorname{det} J_{F_{t}}\left(\begin{array}{l}
x_{0} \\
y_{0}
\end{array}\right) & =\eta(t)^{2} \zeta(t)+\xi(t)^{2} \eta(t) f^{\prime}\left(x_{0}\right)+\xi(t)^{2} \zeta(t)-\xi(t)^{2} \eta(t) f^{\prime}\left(x_{0}\right) \\
& =\left(\eta(t)^{2}+\xi(t)^{2}\right) \zeta(t)=j^{t}>0 \text { for all } t \in[0,1]
\end{aligned}
$$

Thus $F_{t}$ is indeed a diffeomorphism for each $t \in[0,1]$. The fact that $F_{0}$ coincides with the identity map and that $F_{1}$ is identical to $T$ can be easily checked by inserting the values of $\eta$ and $\xi$ at the endpoints into the expression (5.5).

Finally we have to show that $\dot{F}_{t}\left(\begin{array}{l}x_{0} \\ y_{0}\end{array}\right)$ is a continuous function of $t \in[0,1]$ for all points in the plane. To this end we differentiate Eq. (5.5) with respect to $t$.

$$
\dot{F}_{t}\left(\begin{array}{l}
x_{0} \\
y_{0}
\end{array}\right)=\left(\begin{array}{c}
{[\dot{\eta}(t) \zeta(t)+\eta(t) \dot{\xi}(t)] x_{0}+2 \dot{\xi}(t) \xi(t) f\left(x_{0}\right)+\dot{\xi}(t) y_{0}} \\
-[\dot{\xi}(t) \zeta(t)+\xi(t) \dot{\zeta}(t)] x_{0}+[\dot{\xi}(t) \eta(t)+\xi(t) \dot{\eta}(t)] f\left(x_{0}\right)+\dot{\eta}(t) y_{0}
\end{array}\right) .
$$


Since $\xi, \eta, \zeta \in \mathscr{C}^{1}([0,1])$ we know that $\dot{F}\left(\begin{array}{l}x_{0} \\ y_{0}\end{array}\right)$ is continuous in the interior of the interval. According to Eq. (3.5) we still have to show that

$$
\lim _{t \rightarrow 1-} \dot{F}_{t}\left(\begin{array}{c}
x_{0} \\
y_{0}
\end{array}\right)=\lim _{t \rightarrow 0_{+}} \dot{F}_{t}\left(T\left(\begin{array}{l}
x_{0} \\
y_{0}
\end{array}\right)\right) \text {. }
$$

Performing the limit of the left-hand side of Eq. (5.9) for the expression (5.8) and taking into account the conditions (5.4) we get:

$$
\lim _{t \rightarrow 1-} \dot{F}_{t}\left(\begin{array}{l}
x_{0} \\
y_{0}
\end{array}\right)=\left(\begin{array}{c}
0 \\
-\dot{\zeta}(1) x_{0}
\end{array}\right)
$$

for the right-hand side of Eq. (5.9) we obtain with Eq. (5.1):

$$
\lim _{t \rightarrow 0_{+}} \dot{F}_{t}\left(T\left(\begin{array}{l}
x_{0} \\
y_{0}
\end{array}\right)\right)=\left(\begin{array}{c}
0 \\
-j \ln j \cdot x_{0}
\end{array}\right) \text {. }
$$

The equality of (5.10) and (5.11) follows from

$$
\dot{\zeta}(t)=j^{t}\left[\ln j\left(\xi(t)^{2}+\eta(t)^{2}\right)-2(\xi(t) \dot{\xi}(t)+\eta(t) \dot{\eta}(t))\right] \cdot\left(\xi(t)^{2}+\eta(t)^{2}\right)^{-2}
$$

for $t$ going to 1 .

For an explicit realization we have chosen:

$$
\xi(t)=(3-2 t) t^{2}, \quad \eta(t)=1+\ln j \cdot t-(2 \ln j+3) t^{2}+(2+\ln j) t^{3} .
$$

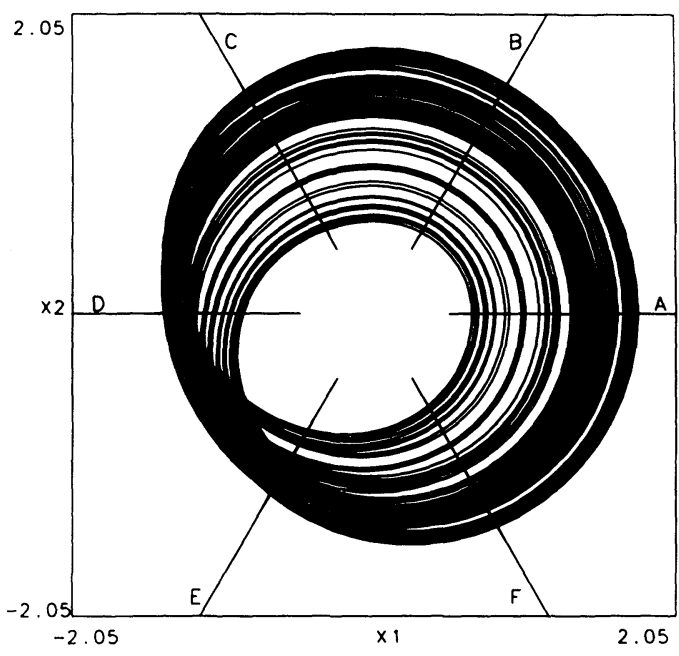

Fig. 1. Projection of the solution $\left(\begin{array}{l}x(t) \\ y(t) \\ z(t)\end{array}\right)$ of Eq. (6.1) for the differential equation (6.3) in a chaotic parameter regime of the Hénon-system $(5.1)(a=2.1, j=0.3)$ onto the $\left(x_{1}, x_{2}\right)$-plane, where: $x_{1}(t)$ $=(x(t)+1) \cos 2 \pi t, x_{2}(t)=(x(t)+1) \sin 2 \pi t$. Note the similarity to the simple Rössler-attractor [12]. The lines $A, B, \ldots, F$ indicate Poincaré-sections at successive angles 


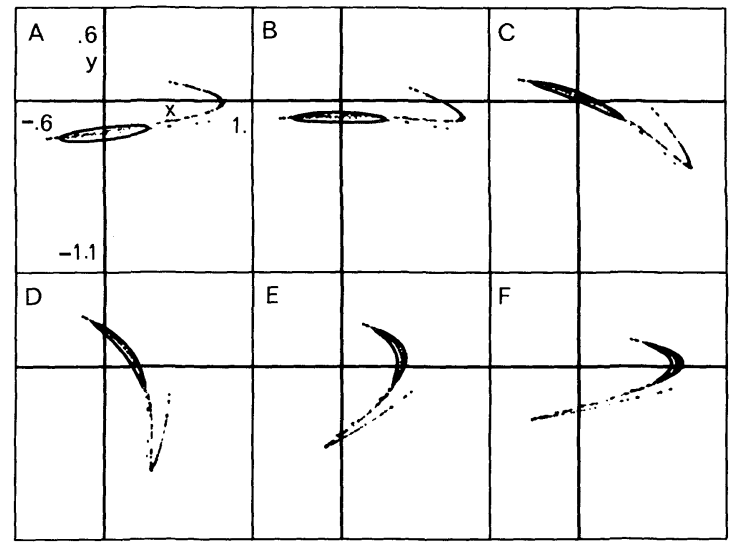

Fig. 2. Poincaré-cross-sections of the suspension of Fig. 1 at successive angles denoted by $A, B, \ldots, F$. In order to illustrate the folding-, stretching-, and construction-process, we also have computed the trajectories through an ellipse in a neighborhood of the attractor

The corresponding suspension of the Hénon-system can be visualized in Fig. 1. We have also produced a $16 \mathrm{~mm}$ film showing the dynamics of the folding processes generated by this suspension. In the same way as seen in Fig. 2, we have computed a series of Poincaré-sections of the attractor for subsequent times. The dynamics are visually equivalent to the dynamics generated by the differential equation of a driven and damped pendulum like the Duffing-oscillator [10]. In the movie one can clearly see how the fractal structure of the chaotic attractor arises. (The same technique has been previously used by Crutchfield et al. [11] to illustrate the folding process in different chaotic flows.)

\section{Autonomous Differential Equations from the Suspension of the Entire Cremona-Transformations}

In the previous section we constructed a smooth suspension for the diffeomorphism (5.1). Thus we can consider Eq. (5.5) as a solution of the differential-equation (5.8). However Eq. (5.8) represents a non-autonomous system since it depends on the initial values $x_{0}, y_{0}$ and not on the actual position at time $t$ which is given by:

$$
\left(\begin{array}{l}
x(t) \\
y(t) \\
z(t)
\end{array}\right)=\left(\begin{array}{l}
F_{t}\left(\begin{array}{l}
x_{0} \\
y_{0}
\end{array}\right) \\
\omega t+t_{0}
\end{array}\right),
$$

where in our case $\omega=1$ and $t_{0}=0$. In order to transform Eq. (5.8) into an autonomous differential equation, we first have to express the initial values $x_{0}, y_{0}$ as a function of $x(t), y(t)$. After straightforward but somewhat lengthy calculations we obtain:

$$
\begin{aligned}
& x_{0}(x(t), y(t))=j^{-t}(\eta(t) x(t)-\xi(t) y(t)), \\
& y_{0}(x(t), y(t))=\frac{\xi(t) x(t)+\eta(t) y(t)}{\xi(t)^{2}+\eta(t)^{2}}-\xi(t) f\left(x_{0}\right) .
\end{aligned}
$$


When we now insert the initial conditions of Eq. (6.2) into Eq. (5.8), we can express $\dot{x}(t), \dot{y}(t)$, and $\dot{z}(t)$ as a function of $x(t), y(t)$, and $z(t)$, and thus have made the differential equations autonomous. They are given by:

$$
\begin{aligned}
& \dot{x}(t)=h(\xi(t), \eta(t)) x(t)+g(\xi(t), \eta(t)) y(t)+\xi(t) \dot{\xi}(t) f\left(x_{0}(x(t), y(t))\right), \\
& \dot{y}(t)=g(\eta(t), \xi(t)) x(t)+h(\eta(t), \xi(t)) y(t)+\dot{\xi}(t) \eta(t) f\left(x_{0}(x(t), y(t))\right), \\
& \dot{z}(t)=\omega,
\end{aligned}
$$

where $x_{0}(x(t), y(t))$ is taken from Eq. (6.2) and the functions $g$ and $h$ are given by:

$$
\begin{aligned}
& g(\xi, \eta)=\frac{1}{\xi^{2}+\eta^{2}}\left\{\dot{\xi} \eta-\xi \dot{\eta}-\xi \eta\left(\ln j-2 \frac{\xi \dot{\xi}+\eta \dot{\eta}}{\xi^{2}+\eta^{2}}\right)\right\}, \\
& h(\xi, \eta)=\frac{1}{\xi^{2}+\eta^{2}}\left\{(\dot{\xi} \xi+\dot{\eta} \eta) \frac{\xi^{2}-\eta^{2}}{\xi^{2}+\eta^{2}}+\eta^{2} \ln j\right\} .
\end{aligned}
$$

Here we have omitted the arguments where no confusion was possible. We see that the only nonlinearity comes indeed from the function $f$ of Eq. (5.1). The main complexity, however, is contained in the $t$ - (or $z$-) dependence of the coefficient functions $g$ and $h$. It would be interesting to see whether it is possible to express $g$ and $h$ as functions of $x(t)$ and $y(t)$ alone. Finally we will show that this autonomous O.D.E. (6.3) in fact represents a driven and damped oscillator with constant dissipation. For this purpose we evaluate the divergence of the vector field in Eq. (6.3). It is given by:

$$
\begin{aligned}
\operatorname{div}\left(\begin{array}{c}
\dot{x} \\
\dot{y} \\
\dot{z}
\end{array}\right) & =\frac{\partial \dot{x}}{\partial x}+\frac{\partial \dot{y}}{\partial y}+\frac{\partial \dot{z}}{\partial z} \\
& =h(\xi, \eta)+\xi \dot{\xi} f^{\prime}\left(x_{0}\right) \cdot j^{-t} \cdot \eta+h(\eta, \xi)+\dot{\xi} \eta f^{\prime}\left(x_{0}\right)\left(-\xi j^{-t}\right) \\
& =h(\xi, \eta)+h(\eta, \xi) .
\end{aligned}
$$

When we now insert the explicit form of $h(\xi, \eta)$ from Eq. (6.4) we get:

$$
\operatorname{div}\left(\begin{array}{c}
\dot{x} \\
\dot{y} \\
\dot{z}
\end{array}\right)=\frac{1}{\xi^{2}+\eta^{2}}\left\{\frac{\dot{\xi} \xi+\dot{\eta} \eta}{\xi^{2}+\eta^{2}}\left(\xi^{2}-\eta^{2}+\eta^{2}-\xi^{2}\right)+\ln j\left(\eta^{2}+\xi^{2}\right)\right\}=\ln j .
$$

This is exactly the "damping constant" which we would expect from Eqs. (5.2) and (5.7).

\section{Conclusion}

We have constructed an explicit solution for a class of chaotic autonomous ordinary differential equations, which is expressed by analytical functions and iterates of a diffeomorphism. The method we have used is based on the notion of smooth suspensions. We have also made a concrete connection between diffeomorphisms in the plane and continuous flows in three-dimensional space. Our emphasis was laid on maps with constant Jacobian and, correspondingly flows 
with a position-independent divergence. These cases correspond to a constant damping term in the equations for a driven and damped oscillator. Thus we have constructed a way to study chaotic dynamics of continuous systems by calculating analytical maps instead of numerically integrating the differential equations.

Acknowledgements. One of us (G.M-K.) would like to thank H. Bunz, K. Marx, and P. Lomdahl for their help with the computer graphics. He is also grateful to Emily Stone and Jim Keeler for their help with the language problems.

\section{References}

1. Collet, P., Eckmann, J.-P.: Iterated maps on the interval as dynamical systems. Boston: Birkhäuser 1980

2. Eckmann, J.-P.: Roads to turbulence in dissipative dynamical systems. Rev. Mod. Phys. 53, 643 (1981);

Ruelle, D., Takens, F.: On the nature of turbulence. Commun. Math. Phys. 20, 167 (1971); Pomeau, Y., Manneville, P.: Intermittent transition to turbulence in dissipative dynamical systems. Commun. Math. Phys. 77, 789 (1980)

3. Feigenbaum, M.: Quantitative universality for a class of nonlinear transformations. J. Stat. Phys. 19, 25 (1978)

4. Grossmann, S.: Private communication

5. See, e.g., Haken, H.: Advanced synergetics. Berlin, Heidelberg, New York: Springer 1983

6. Gonzalez, O.L., Piro, O.: Chaos in a nonlinear driven oscillator with exact solution. Phys. Rev. Lett. 50, 870 (1983)

7. Farmer, J.D., Crutchfield, J.P., Froehling, H., Packard, N., Shaw, R.: Power spectra and mixing properties of strange attractors. Proc. of the 1979 Conf. on Nonlin. Dyn. Helleman, R.H.G. (ed.). Ann. N.Y. Acad. Sci. 375, 353 (1980)

8. Smale, S.: Bull. Am. Math. Soc. 73, 747 (1967)

9. Hénon, M.: A two-dimensional mapping with a strange attractor. Commun. Math. Phys. 50, 69 (1976)

10. Bunz, H.: private communication

11. Crutchfield, J.P., Farmer, J.D., Packard, N., Shaw, R.: Mixing properties of chaotic attractors. $16 \mathrm{~mm}$ film

12. Rössler, O.E.: An equation for continuous chaos. Phys. Lett. 57A, 397 (1976)

13. Mayer-Kress, G.: Zur Persistenz von Chaos und Ordnung in nichtlinearen dynamischen Systemen. Ph. D. thesis, Universität Stuttgart 1984

Communicated by O. E. Lanford

Received September 19, 1984

Note added in proof. During the careful and extensive review of this paper by the editor one of us (G. M-K.) was able to generalize our results to $C^{1}$-suspensions of diffeomorphisms on the 2-torus (Mayer-Kress, G.: Autonomous Differential Equations for the Hénon Map and other TwoDimensional Diffeomorphisms. In: Perspectives in Nonlinear Dynamics. Shlesinger, M.F., Cawley, R., Saenz, A.W., Zachary, W. (eds.). Singapore: World Scientific 1986). Furthermore we have been informed about independent constructions of Lipshitz suspensions by P. Channell (J. Math. Phys. 24, 823 (1983)) and D. Elliot (forthcoming report). 